Paper

\title{
Quality Estimation Method for Fractal Compressed Images
}

\begin{abstract}
Megumi Takezawa (member) ${ }^{\dagger}$, Hirofumi Sanada ${ }^{\dagger}$, Miki Haseyama (member) ${ }^{\dagger \dagger}$
Abstract A method for estimating the quality of images compressed by fractal image compression is presented in this paper. Fractal image compression based on an iterated function system is one of the compression techniques for digital images. It utilizes the self-similarity of images and achieves high image-compression performance. However, fractal image compression is currently not being in widespread use because it does not necessarily provide high-quality compressed images. We cannot determine whether a given image is unsuitable for fractal image compression without encoding it. Therefore, in this paper, we propose a new criterion for estimating the suitability of fractal image compression for a given image. By using the proposed criterion, we can estimate the quality of the compressed image in a short time without actually encoding the image.
\end{abstract}

Key words: fractal image compression, self-similarity, image quality, fractal dimension

\section{Introduction}

Fractal image compression ${ }^{\left.1)^{2) 3}\right)}$ based on an iterated function system (IFS), one of the compression techniques for digital images, compresses images by utilizing their self-similarity. High compression performance can be achieved by fractal image compression by storing the coefficients of the IFS instead of image pixels. Moreover, in addition to the high compression performance, fractal image compression has the advantages of enabling images to be decoded in a few seconds and at an arbitrary resolution. However, one of the drawbacks of fractal image coding is that the quality of some compressed images is not sufficient for practical applications. Additionally, what is more inconvenient is that we cannot determine whether the quality of the compressed image for a given image will be low unless we actually encode and decode it, which takes a long time. This problem must be solved in order for fractal image coding to become a practical technique. We therefore decided to try to solve this problem by the following steps.

(i) A new criterion for determining the suitability of fractal image compression for a given image is

Received October 10, 2012; Revised December 7, 2012; Accepted December 27, 2012

$\dagger$ The authors are with the Department of Frontier Information Engineering, Hokkaido Institute of Technology (7-15 Maeda, Teine-ku, Sapporo 006-8585, Japan.)

$\dagger \dagger$ The author is with the School of Information Science and Technology, Hokkaido University

(N-14 W-9 Kita-ku, Sapporo 060-0814, Japan.) given.

(ii) Based on the criterion, we determine how to apply fractal image compression to each image. If its suitability is high, fractal image compression is simply applied to the given image. If its suitability is low, other coding techniques, such as the hybrid coding technique of fractal image compression and a wavelet compression technique, are applied.

A criterion for determining the suitability of fractal image compression has not been given in any previous papers. In addition, such an approach, which changes the way of applying fractal image compression according to the characteristics of each image, has not been presented thus far. Our approach enables the possibility of using fractal image compression in combination with standard coding techniques, such as Joint Photographic Experts Group (JPEG) coding and wavelet coding, and extends the use of fractal image compression. In this paper, criterion(i) is proposed. By using this criterion, we can determine whether a given image is suitable for fractal image compression, and then we can apply an effective compression technique to each image.

\section{Fractal image compression}

The basic procedure for fractal image compression ${ }^{1)}$ 2)3) is shown in Fig. 1. The procedure is executed as follows:

(i) A given image $I$ of size $W_{1} \times W_{2}$ is partitioned into nonoverlapping blocks (hereafter called range blocks) of size $B \times B$ and into arbitrarily lo- 
cated blocks (hereafter called domain blocks) of size $2 B \times 2 B$. The range blocks are numbered from 1 to $M\left(=W_{1} / B \times W_{2} / B\right)$ and denoted by $R_{i}(i=1,2, \cdots, M)$. Similarly, the domain blocks are numbered from 1 to $N\left(=\left(W_{1}-2 B+1\right) \times\left(W_{2}-\right.\right.$ $2 B+1))$ and denoted by $D_{j}(j=1,2, \cdots, N)$.

(ii) Each domain block $D_{j}$ is contracted by the affine transformation $\tau_{i j}$ defined as follows:

$$
\begin{gathered}
\left(\begin{array}{c}
\tilde{p} \\
\tilde{q} \\
\tilde{f}(\tilde{p}, \tilde{q})
\end{array}\right)=\tau_{i j}\left(\begin{array}{c}
p \\
q \\
f(p, q)
\end{array}\right) \\
=\left(\begin{array}{ccc}
\frac{1}{2} \cos \theta & -\frac{1}{2} \sin \theta \times \lambda & 0 \\
\frac{1}{2} \sin \theta & \frac{1}{2} \cos \theta \times \lambda & 0 \\
0 & 0 & \alpha
\end{array}\right)\left(\begin{array}{c}
p \\
q \\
f(p, q)
\end{array}\right) \\
+\left(\begin{array}{c}
g \\
h \\
\beta
\end{array}\right), \quad(1)
\end{gathered}
$$

where $\theta \in\left\{0^{\circ}, 90^{\circ}, 180^{\circ}, 270^{\circ}\right\}$ and $\lambda \in\{1,-1\}$. $(p, q)$ and $(\tilde{p}, \tilde{q})$ are the coordinates of the pixels in the domain block $D_{j}$ and the obtained block $\tau_{i j}\left(D_{j}\right)$, respectively, and $f(p, q)$ and $\tilde{f}(\tilde{p}, \tilde{q})$ are their pixel values. The parameters $g, h \in\{0, B-1\}$ are offsets for the coordinates. The parameters $\alpha$ and $\beta$ are called the scaling coefficient and offset, respectively.

(iii) For each range block $R_{i}$, we find the best contracted domain block $D_{i}^{\text {opt }}$ among the contracted domain blocks obtained by using various $\theta, \lambda, \alpha$ and $\beta$ values in Eq. (1). The best contracted domain block $D_{i}^{\text {opt }}$ satisfies the following equation:

$$
d\left(R_{i}, D_{i}^{\mathrm{opt}}\right)=\min _{\theta, \lambda, \alpha, \beta, j} d\left(R_{i}, \tau_{i j}\left(D_{j}\right)\right) .
$$

In the above equation, the distortion measure $d(x, y)$ is the mean square error (MSE) between $x$ and $y$.

(iv) For each range block, the parameters that provide the best contracted domain block (hereafter called IFS parameters) are encoded. The IFS parameters are the parameters $\left(\theta_{i}^{\mathrm{opt}}, \lambda_{i}^{\mathrm{opt}}, \alpha_{i}^{\mathrm{opt}}, \beta_{i}^{\mathrm{opt}}\right)$ of the best affine transformation and the parameters $\left(x_{i}^{\mathrm{opt}}, y_{i}^{\mathrm{opt}}\right)$ indicating the location of the best matched domain block.

\section{Self-similarity of images}

Self-similarity means that a small portion of an object can be viewed as a microcosm of the larger object, and an object that has self-similarity is called a fractal ${ }^{4)}$.

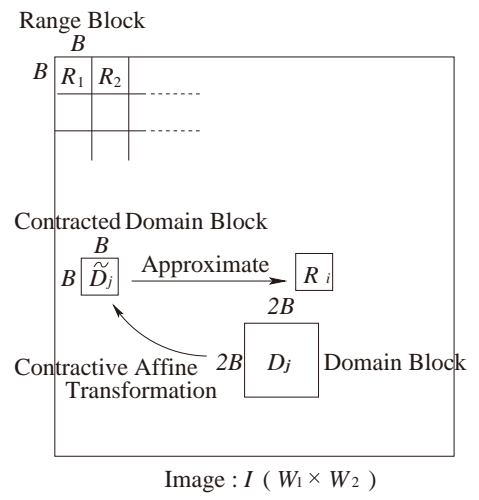

Fig. 1 Procedure of fractal image compression.

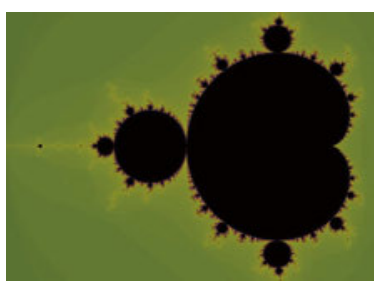

Fig. 2 Mandelbrot set.

One of the simplest examples of a fractal is the Mandelbrot set shown in Fig. 2. Generally, natural images do not have exact self-similarity but have approximate selfsimilarity. Therefore, fractal image compression compresses images by using the approximate self-similarity of images.

As stated above, natural images generally do not have exact self-similarity. However, if they have exact self-similarity, their fractal compressed images will be close to the original ones. Hence, it is conceivable that the level of self-similarity of an image appears to be closely related to the quality of the fractal compressed image. Therefore, in this paper, we use the level of selfsimilarity as one feature to estimate the suitability of fractal image compression. In the following subsections, we present a criterion for the level of self-similarity of an image based on the process of calculating the fractal dimension of the image. In the first subsection, fractal dimension is explained, the criterion for the level of self-similarity is given in the second subsection, and the validity of the criterion is verified in the third subsection.

\section{1 Fractal dimension}

Fractal dimension represents the degree of complexity of objects. The dimension of an object that can be drawn as a line is between 1 and 2, and the dimension of an object created from a shape on a plane is between 2 and 3 . In most cases, the fractal dimensions of natural objects are nonintegers. Unusual cases in which the dimension of an object is the integer 1,2 or 3 are 


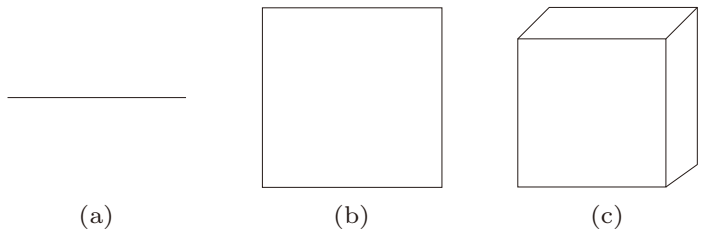

Fig. 3 Fractal dimensions: (a) 1.0; (b) 2.0; (c) 3.0.

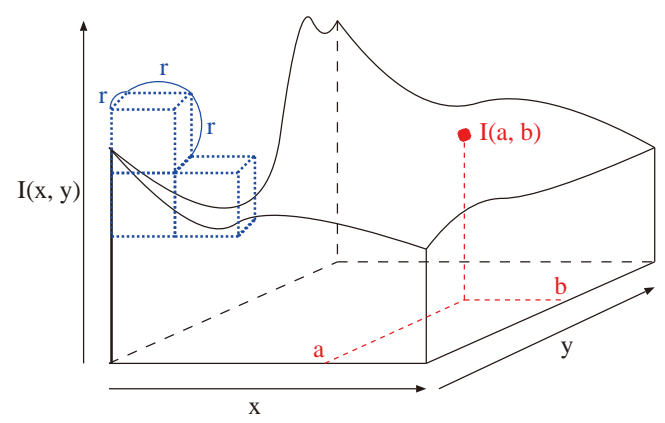

Fig. 4 How to calculate fractal dimension of image by box-counting method.

shown in Figs. 3(a), (b), and (c), respectively. Many methods for calculating the fractal dimension of an object have been proposed ${ }^{5)-8)}$. In this paper, we use the box-counting method $^{5)}$. In the following, we describe the basic procedure for calculating the box-counting dimension of gray-scale images with reference to Fig. 4 .

(i) In a given image, the coordinates of the pixels are denoted by $(x, y)$ and their intensity values are $I(x, y)$.

(ii) The intensity surface $(x, y, I(x, y))$ is covered with cubes of size $r \times r \times r\left(r=1, \cdots, r_{\max }\right)$, and the number of required cubes is denoted by $N(r)$.

(iii) $\quad N(r), r$, and the fractal dimension $D$ satisfy the following equation:

$$
N(r) \propto r^{-D}
$$

Therefore, the fractal dimension can be calculated from the slope of the plot of $\log r$ versus $\log N(r)$. Because the computational cost of step (ii) increases with increasing size of the cubes, we used values of $r=3,5, \cdots, 41^{6)}$.

\section{2 Level of self-similarity}

In the case of natural images, the obtained plots of $\log r$ versus $\log N(r)$ discussed in Sect.3.1 (iii) do not give straight lines because they do not have exact selfsimilarity. As a reference, Figs. 5(a) and (b) show the plots of $\log r$ versus $\log N(r)$ obtained for the two natural images in Figs. 6(a) and (b). Therefore, the fractal dimension is estimated from the slope of the best-fit regression line to the data points $(\log r, \log N(r))$. On the other hand, if an image has exact self-similarity, its

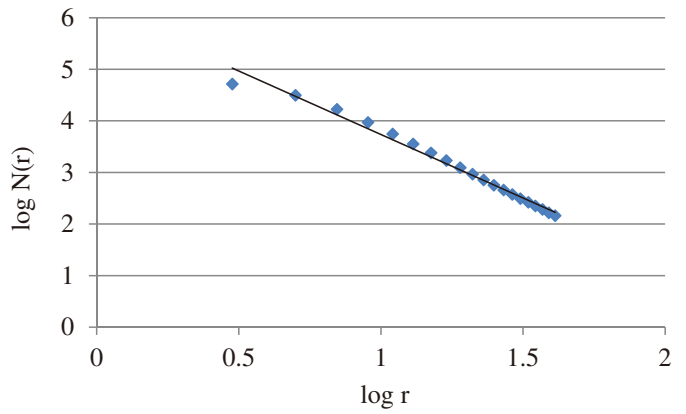

(a)

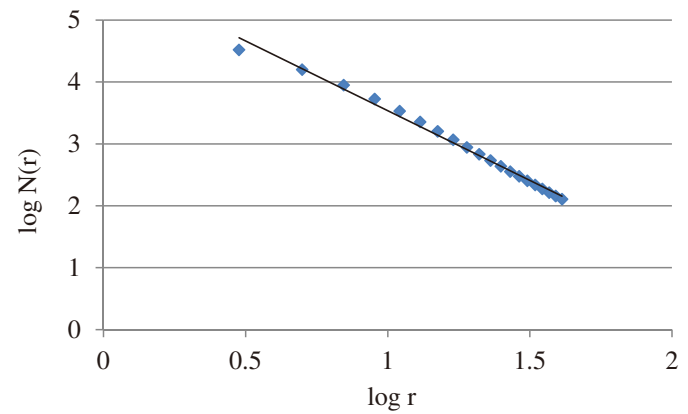

(b)

Fig. 5 Relationship between $r$ and $N(r)$ : (a) Fig. 6(a); (b) Fig. 6(b).

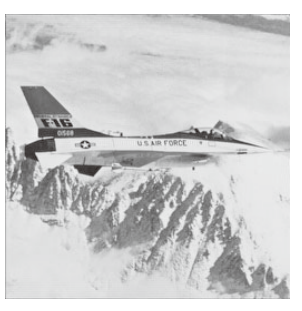

(a)

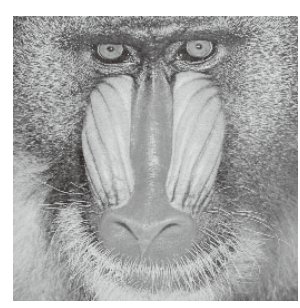

(b)
Fig. 6 Natural images: (a) airplane; (b) mandrill.

plot is a straight line. Hence, from this fact, the level of self-similarity is represented by the goodness of fit to the regression line ${ }^{9)}$. Thus, in this paper, we utilize the correlation coefficient between actual measured values and values predicted from the regression line as a criterion for the level of self-similarity of an image.

\subsection{Relationship between self-similarity of original image and quality of compressed image.}

To determine the relationship between the selfsimilarity of an original image and the quality of an image compressed by fractal image compression, we calculate the level of self-similarity using the criterion explained in Sect.3. 2 for the ten still images with a size of $256 \times 256$ pixels and an 8-bit gray level shown in Fig. 7. The calculated level of self-similarity and the actual mean square errors (MSEs) of a collage that is obtained by applying IFS mapping to an original image are shown in Fig. 8. Here, we use the fractal image 


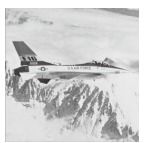

(a)

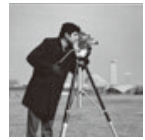

(f)

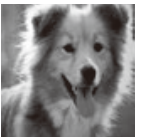

(g)

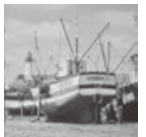

(c)

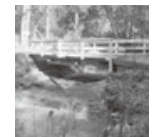

(d)

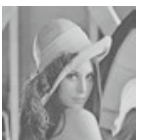

(h)

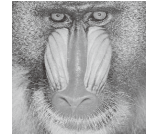

(i)

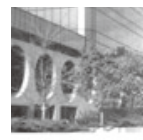

(e)

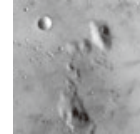

(j) (d)bridge, (e)building, (f)cameraman, (g)collie, (h)lena, and (i)moon).

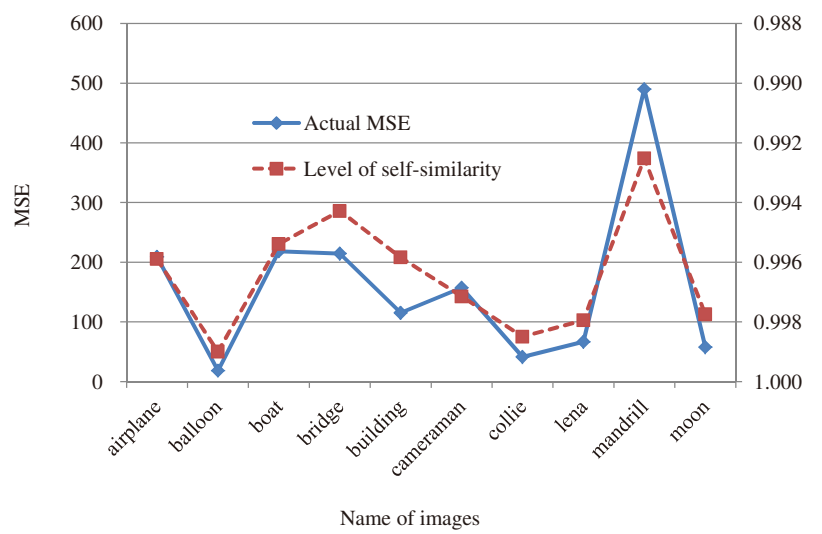

Fig. 8 Self-similarity of original image and MSE of compressed image.

compression introduced in Sect. 2, and the range block size is $8 \times 8$ pixels.

In this figure, we can see that fluctuations in selfsimilarity correlate closely with changes in MSE. Hence, the results indicate that the level of self-similarity of an image is closely related to the quality of the compressed image. However, a difference in MSE is observed between the compressed images even if their original images have the same level of self-similarity (for example, boat and building). Therefore, it is difficult for us to estimate the compressed image quality from only its self-similarity and determine whether fractal image compression is suitable for the given image. Hence, an effective criterion based on not only self-similarity but also other features of the image is proposed in this paper. By using the proposed criterion, for a given image, we can estimate the quality of the compressed image accurately and thus determine the suitability of fractal image compression for it.

\section{Criterion for suitability of fractal im- age compression}

As stated in Sect. 3. 3, the level of self-similarity of an image is closely related to the quality of the image com- pressed by fractal image compression. However, that quality cannot be estimated from only the level of selfsimilarity. Therefore, we try to estimate the quality of the fractal compressed images by linearly transforming some features, namely, the fractal dimension, the level of self-similarity, the average, the variance and the correlation coefficient between neighboring pixels.

To determine the relationship between the quality of an image compressed by fractal image compression and its image features, we calculate the five features for the still images shown in Fig. 7. The calculated fractal dimension, the level of self-similarity, the average, the variance, and the correlation coefficient between neighboring pixels are shown in Table $\mathbf{1}$ for the test images.

Furthermore, to determine the strength of the relationship between the quality of the compressed image and the five image features, we show the actual MSEs of the compressed images and the MSEs estimated from the image feature values in Fig. 9(a).

In this figure, the MSEs of the actual compressed images are shown as (i), and (ii), (iii), (iv), (v), and (vi) are the MSEs estimated from the fractal dimension, the level of self-similarity, the average, the variance, and the correlation coefficient, respectively. Here, we use simple regression analysis to estimate the MSEs of the compressed images. According to this figure, it is difficult to estimate the MSEs of the compressed images using only one feature.

Therefore, we selected the best subset of features and estimated the MSEs by stepwise multiple regression analysis. The selected features are the fractal dimension, the level of self-similarity and the correlation coefficient. We estimated the MSEs of the compressed images using the following equation:

$$
f=\alpha d+\beta s+\gamma c+\delta
$$

where $d$ is the fractal dimension, $s$ is the level of selfsimilarity calculated as described in Sect.3.2, $c$ is the correlation coefficient and $\alpha, \beta, \gamma$ and $\delta$ are constants. The values of $\alpha, \beta, \gamma$ and $\delta$ were determined by multiple regression analysis. The MSEs estimated from the values of these features are shown as (ii) in Fig. 9(b). Here, the coefficient of determination between the actual MSEs and the estimated MSEs was 0.979. From this result, we can accurately estimate the MSEs when using these features. Moreover, we evaluated the time required to determine the quality of a compressed image. The total CPU time actually used to encode and decode the image was 583 seconds. In contrast, the 
Table 1 Feature values of original images: fractal dimension, level of self-similarity, average, variance, and correlation coefficient between neighboring pixels.

\begin{tabular}{|c|c|c|c|c|c|}
\hline Images & Fractal dimension & Level of self-similarity & Average & Variance & Correlation coefficient \\
\hline airplane & 2.25 & 0.996 & 178.7 & 2114.3 & 0.925 \\
balloon & 2.22 & 0.999 & 138.9 & 637.7 & 0.961 \\
boat & 2.24 & 0.995 & 136.2 & 2737.6 & 0.914 \\
bridge & 2.33 & 0.994 & 113.9 & 2823.3 & 0.931 \\
building & 2.27 & 0.996 & 127.8 & 3288.6 & 0.964 \\
cameraman & 2.15 & 0.997 & 118.7 & 3948.3 & 0.935 \\
collie & 2.21 & 0.998 & 94.9 & 3235.8 & 0.985 \\
lena & 2.28 & 0.998 & 73.6 & 1816.5 & 0.973 \\
mandrill & 2.46 & 0.993 & 129.1 & 1747.7 & 0.732 \\
moon & 2.32 & 0.998 & 130.8 & 807.6 & 0.902 \\
\hline
\end{tabular}

CPU time was only 17 seconds for estimating the quality of the compressed image by the proposed method. The proposed method was able to estimate the quality of the compressed image with only $2.9 \%$ of the computational cost of the encoding and decoding process. Furthermore, we experimented with still images with a larger size of $1024 \times 1024$ pixels and an 8-bit gray level. The CPU time of the proposed method was $0.3 \%$ of that required to encode and decode images by fractal image compression. These results verify that we can estimate the quality of a compressed image in a short time and that the proposed method would be more beneficial with higher resolution images.

\section{Conclusion}

Fractal image compression sometimes provides lowquality compressed images, which makes the practical use of fractal image compression difficult. Therefore, we have devised a new approach for applying fractal image compression to images. Our approach is as follows: First, a criterion for the suitability of fractal image compression for a given image is given. Then, from this criterion, we determine how to apply fractal image compression to each image: if its suitability is high, fractal image compression is simply applied to the given image, and if its suitability is low, other coding techniques are applied. Such an approach is novel and will promote the use of fractal image compression. In this paper, we propose a criterion for the suitability of fractal image compression. The results of experiments verify the validity of the proposed criterion.

Further research will focus on methods for applying fractal image compression to high and low-suitability images.

\section{References}

1) M.F. Barnsley, Fractals Everywhere, Academic Press, Boston (1988)

2) A.E. Jacquin, "Image coding based on fractal theory of iterated contractive image transformations," IEEE Trans. Image Process.,

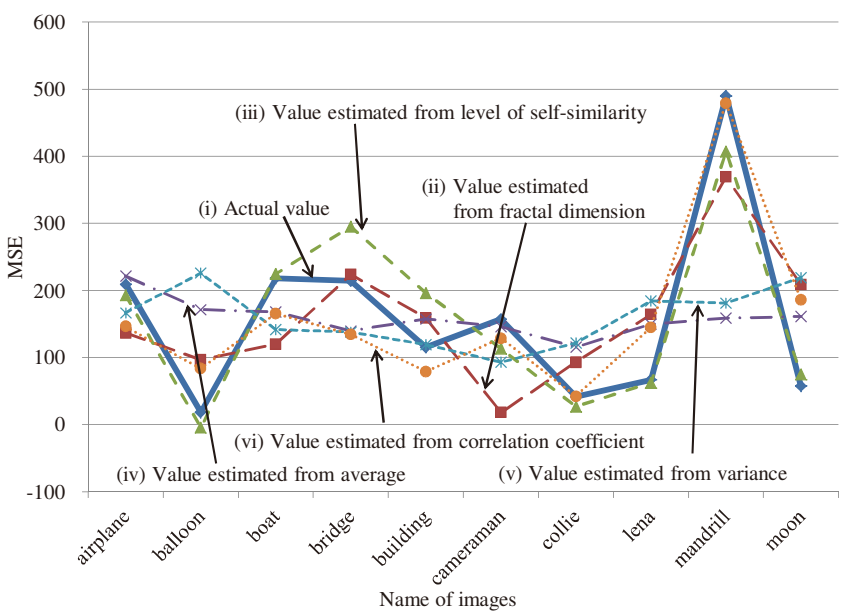

(a)

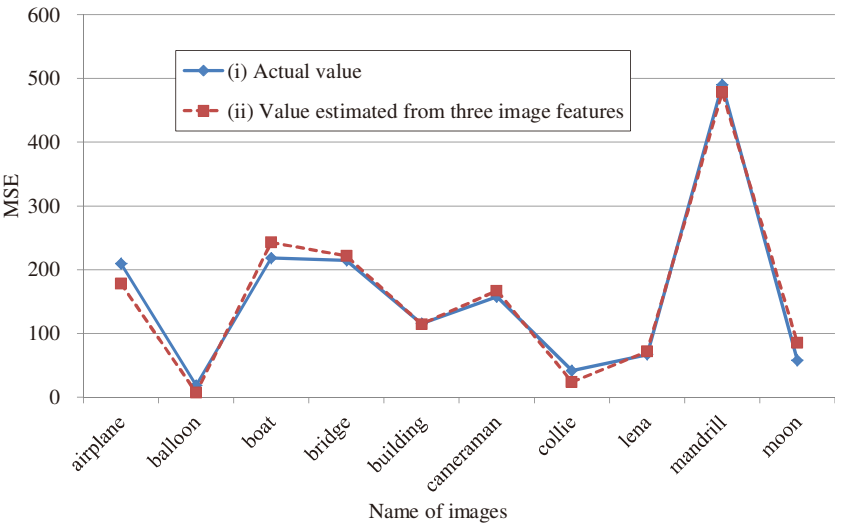

(b)

Fig. 9 Estimated MSEs obtained from image feature values and actual MSEs of compressed images.

vol.1, no.1, pp.18-30 (1992)

3) Y. Fisher, ed., Fractal Image Compression: Theory and Application, Springer-Verlag, New York (1995)

4) M. Mandelbrot, ed., The Fractal Geometry of Nature, W.H. Freeman, New York (1983)

5) R. Voss, "Random fractals: characterization and measurement," Scaling Phenom. in Disordered Syst., pp.1-11 (1986)

6) J.M. Keller, S. Chen, and R. Crownover, "Texture description and segmentation through fractal geometry," Comput. Vision, Graphics Image Process., vol.45, pp.150-166 (1999)

7) S. Peleg, J. Naor, R. Hartly, and D. Avnir, "Multiple resolution texture analysis and classification," IEEE Trans. Pattern Anal. Mach. Intell., vol.PAMI-6, no.4, pp.518-523 (1984)

8) B. Dubuc, "Evaluating the fractal dimension of surfaces," Proc R. Soc. London A, vol.425, pp.115-127 (1989)

9) H. Kaneko, "Fractal feature and texture analysis," Trans. Inst. Electron. Inf. Commun. Eng., vol.70, no.5, pp.964-972 (1987) 
Megumi Takezawa received her B.S., M.S., and Ph.D. degrees in Information Engineering from Hokkaido University, Japan, in 1999, 2001, and 2004, respectively. She is currently an Associate Professor of the Department of Frontier Information Engineering, Hokkaido Institute of Technology. Her research interests include image processing.

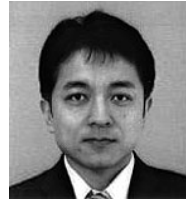

Hirofumi Sanada received his B.S., M.S., and Ph.D. degrees in Electronics from Hokkaido University, Japan, in 1991, 1993, and 1998, respectively. He is currently a Professor of the Department of Frontier Information Engineering, Hokkaido Institute of Technology. His research interests are the development of numerical design techniques for various microwave filters, and image processing.

Miki Haseyama received her B.S., M.S. and Ph.D. degrees in Electronics from Hokkaido University, Japan in 1986, 1988 and 1993, respectively. She joined the Graduate School of Information Science and Technology, Hokkaido University as an associate professor in 1994. She was a visiting associate professor of Washington University, USA from 2005 to 2006. She is currently a professor in the Graduate School of Information Science and Technology, Hokkaido University. Her research interests include image and video processing and its development into semantic analysis. She is a member of the IEEE, IEICE, Institute of Image Information and Television Engineers (ITE) and Acoustical Society of Japan (ASJ). 